\title{
Nefrectomía parcial laparoscópica: Técnica y resultados
}

\author{
Colombo JR Jr, Gill IS. \\ Sección de Cirugía Laparoscópica y Robótica, Glickman Urological Institute, Cleveland Clinic Foundation, \\ Cleveland, OH, EE.UU.
}

Actas Urol Esp 2006: 30 (5) 501-505

\section{RESUMEN \\ NEFRECTOMÍA PARCIAL LAPAROSCÓPICA: TÉCNICA Y RESULTADOS}

La indicación de la Nefrectomía Parcial Laparoscópica (NPL) ha evolucionado considerablemente y la técnica se está convirtiendo en una opción establecida en nuestro centro. En los últimos 5 años, el autor principal ha realizado más de 450 nefrectomías parciales laparoscópicas en la Clínica Cleveland. Presentamos seguidamene nuestra técnica y revisamos los datos actuales y los resultados oncológicos de la NPL.

Palabras clave: Nefrectomía parcial. Laparoscopia. Resultados.

\section{ABSTRACT}

LAPAROSCOPIC PARTIAL NEPHRECTOMY: TECHNIQUE AND OUTCOMES

The indication of laparoscopic partial nephrectomy (LPN) has evolved considerably, and the technique is approaching established status at our institution. Over the past 5 years, the senior author has performed more than 450 laparoscopic partial nephrectomies at the Cleveland Clinic. Herein we present our current technique, review contemporary data and oncological outcomes of LPN.

Keywords: Partial nephrectomy. Laparoscopy. Outcomes.

$\mathrm{L}^{2}$ a nefrectomía parcial laparoscópica (NPL) fue descrita inicialmente en $1993^{1,2}$. El uso extendido de las técnicas radiológicas contemporáneas ha conducido a un aumento de la detección de tumores renales pequeños incidentales y muchos centros han publicado sus experiencias con la $\mathrm{NPL}^{3-9}$. Al principio se seleccionaron tumores pequeños, exofiticos, para la NPL y, con el aumento de la experiencia, se han tratado tumores más grandes y más profundos mediante esta técni$\mathrm{ca}^{4,5,10}$. La hemostasia del parénquima renal, la reparación impermeable al agua de los cálices mediante suturas después de la escisión tumoral y la escisión tumoral completa son los objetivos principales de la NPL, que reproduce de esta forma la nefrectomía parcial abierta. Para reducir al mínimo los problemas técnicos, recientemente se han explorado nuevas técnicas y tecnologías. En este artículo describimos nuestra técnica actual y los resultados de la nefrectomía parcial laparoscópica.

\section{TÉCNICA}

Con nuestra técnica intentamos reproducir los principios oncológicos y reconstructivos establecidos e inherentes a la nefrectomía parcial abierta $^{10}$. La selección del abordaje laparoscópico depende de la localización del tumor, de modo que los tumores posteriores o posterolaterales se abordan retroperitoneoscópicamente, mientras que los tumores anteriores, anterolaterales o laterales se tratan con un abordaje transperitoneal. Las pruebas de imagen preoperatorias con tomografia computarizada en 3D con reconstrucción en vídeo del volumen y la ecografía intraoperatoria en tiempo real proporcionan al cirujano información detallada que facilita el procedimiento, evitando las complicaciones y los márgenes positivos.

Todos los pacientes se someten a la colocación cistoscópica de un catéter ureteral de calibre $5 \mathrm{fr}$ de extremo abierto, situado en la pelvis renal. Se 
fija tinción de índigo-carmín diluida al catéter ureteral y se usa una inyección retrógrada para confirmar la entrada en el sistema colector y el cierre impermeable. El catéter se retira 24 horas después del procedimiento.

\section{Abordaje transperitoneal}

Típicamente, se afianza al paciente a la mesa en una posición de 45 a 60 grados lateral y se emplea un abordaje transperitoneal de 4 ó 5 puertos. Se identifican el uréter y la vena gonadal y se retraen lateralmente. Se realiza la disección a lo largo del músculo psoas y se diseca el hilio renal en bloque. Se diseca la fascia de Gerota separándola del riñón, conservando la grasa perirrenal en contacto con el tumor. Luego se coloca una pinza laparoscópica de Satinsky para pinzar el hilio. Hay que tener cuidado de no romper ningún vaso lumbar en el hilio al aplicar la pinza. Los tumores pequeños, superficiales, completamente exofiticos pueden tratarse sin pinzamiento hiliar $^{11}$.

Se introduce una sonda flexible Doppler color de ecografía a través de un puerto de 10/12 mm y se coloca en contacto directo con la superficie del riñón. Se obtiene información relativa al tamaño tumoral, la profundidad de la extensión intraparenquimatosa y la distancia respecto al sistema colector. Se marca la cápsula renal circunferencialmente con electrocauterio en gancho $\mathrm{J}$ bajo guía ecográfica manteniendo un margen aproximado de $0,5 \mathrm{~cm}$ del parénquima renal normal alrededor del tumor. Se introducen rodillos de Surgicel (Johnson \& Johnson, New Brunswick, New Jersey) y una sutura con agujas (Suturas de Vicryl n. ${ }^{\circ} 1$ en una aguja CT-X) y se colocan bajando por la gotiera paracólica. Se administran manitol (de 12,5 a $25 \mathrm{~g}$ ) y furosemida (de 10 a $20 \mathrm{mg}$ ) por vía intravenosa. Si se espera que el tiempo de isquemia caliente dure más de 30 minutos, se genera hipotermia renal $^{12}$.

La intensidad de la lesión isquémica renal es directamente proporcional a la duración de la isquemia ${ }^{13}$. Una práctica aceptada durante la cirugía conservadora de parénquima renal ha sido limitar la isquemia caliente a $30 \mathrm{~min}$, utilizándose hipotermia cuando se esperan tiempos prolongados. Se han estudiado diversos métodos para abordar este problema ${ }^{12,14,15}$. En la Clínica Cleveland, se emplea hipotermia con hielo semiderretido sólo cuando se esperan tiempos isquémicos prolongados. Además, se administran hidratación adecuada y manitol para optimizar la perfusión renal y la producción de orina.

Se pinza el hilio y se extirpa el tumor con tijeras frías. Se envía una biopsia de escisión de la base para análisis en cortes congelados y se cierra el sistema colector con una sutura continua de Vicryl 2-0 con una aguja CT-1, si es necesario. Se realiza una inyección del índigo-carmín para confirmar el cierre impermeable del sistema colector. La reparación parenquimatosa se termina usando suturas simples de Vicryl N. ${ }^{\circ} 1$ con una aguja CT-X. Se ponen suturas interrumpidas sobre el rodillo de Surgicel, se pone un clip Hem-o-Lock (Weck Closure System, Research Triangle Park, NC) en la sutura para impedir que se abra y se aplica el sellante biológico FloSeal (Baxter, Mountain View, CA) a la superficie detrás del rodillo. Luego se ata la sutura, con una compresión adecuada. Las suturas se colocan dependiendo de la magnitud de la resección. Se suelta la pinza de Satinsky y se confirma la hemostasia y la revascularización renal completas. Se coloca el tumor en un saco impermeable y se extrae a través de una incisión en el lugar del puerto abdominal inferior mínimamente ampliada. Se coloca un drenaje de Jackson-Pratt en pacientes en los que se haya producido entrada al cáliz y se realiza salida laparoscópica.

\section{Abordaje retroperitoneal}

Con el abordaje retroperitoneal, después de la dilatación con balón y la colocación de 3 puertos (de $12 \mathrm{~mm}$ ), se disecan la arteria y la vena renales para facilitar la aplicación de pinzas bulldog laparoscópicas a cada vaso. Recientemente, hemos pinzado el hilio en bloque usando una pinza de Satinsky posicionada a través de un trócar distinto de $12 \mathrm{~mm}$. Al igual que en el abordaje transperitoneal, se extirpa el tumor y se consiguen reparación parenquimatosa renal y hemostasia, con suturas caliciales si es necesario. Se retira la pinza bulldog de la vena renal inicialmente y luego, de la arteria renal. Se realizan la colocación del catéter de drenaje y la salida. 


\section{RESULTADOS}

Hasta este momento, hemos abordado más de 450 NPL en nuestro centro. Se comparó una cohorte de 100 pacientes sometidos a NPL con un grupo de 100 pacientes sometidos a cirugía abierta conservadora de parénquima renal por un tumor renal único esporádico de $7 \mathrm{~cm}$ o menos en nuestro centro ${ }^{16}$. La mediana de tamaño del tumor fue de 2,8 cm en el grupo laparoscópico, en comparación con $3,3 \mathrm{~cm}$ en el grupo abierto (valor de $\mathrm{p}=0$,005). Comparando ambos grupos, la mediana de tiempo quirúrgico fue de 3 frente a 3,9 h $(\mathrm{p}<0,001)$, la pérdida estimada de sangre fue de 125 frente a $250 \mathrm{ml}(\mathrm{p}<0,001)$; y la media de tiempo de isquemia caliente fue de 28 frente a 18 minutos $(\mathrm{p}<0,001)$. Los pacientes del grupo laparoscópico precisaron menos analgesia postoperatoria y experimentaron una estancia hospitalaria y un período de convalecencia más cortos (valor de $\mathrm{p}<0,001$ para las 3 comparaciones). Aunque la cifra absoluta de complicaciones intraoperatorias fue mayor en el grupo laparoscópico, la frecuencia de complicaciones postoperatorias fue similar ( $9 \%$ frente a $14 \%$; valor de $\mathrm{p}=0,27$ ). Hubo dos márgenes quirúrgicos positivos focales en el grupo laparoscópico y ninguno en el grupo abierto. A los 2 y 3 años de seguimiento, todos los pacientes han permanecido sin enfermedad.

Desai et al. evaluaron el impacto de la oclusión hiliar renal isquémica caliente sobre la función renal en 179 pacientes después de una nefrectomía parcial laparoscópica ${ }^{17}$. La media de duración de la isquemia caliente para el grupo entero fue de 31 minutos (rango de 4-55 min). El estudio no reveló cambios significativos en la creatinina sérica al dividir a los pacientes de acuerdo con la duración de la isquemia caliente, la edad y/o la creatinina sérica basal. En pacientes con un riñón único $(\mathrm{N}=15)$, hubo una elevación transitoria de la creatinina sérica en el período postoperatorio inmediato; sin embargo, la elevación en porcentaje de la creatinina sérica respecto a la basal (media de $1,3 \%$ ) en el último seguimiento (media de 4,8 meses) se aproximó a la cantidad subjetiva del parénquima renal extirpado (media del 29\%). La azotemia preexistente y la edad avanzada aumentaron el riesgo de disfunción renal postoperatoria si el tiempo de isquemia caliente era mayor de 30 minutos.
Guillonneau et al. realizaron retrospectivamente una comparación de la nefrectomía parcial laparoscópica con $(\mathrm{N}=12)$ y $\sin (\mathrm{N}=16)$ pinzamiento hiliar renal ${ }^{18}$. El tamaño tumoral era mayor en el grupo con pinzamiento hiliar renal (2,5 frente a $1,9 \mathrm{~cm}$.). El grupo sin pinzamiento hiliar renal tuvo asociada una pérdida de sangre significativamente mayor (708 $\mathrm{ml}$ frente a $270 \mathrm{ml}, \mathrm{p}=0,014)$ y un tiempo quirúrgico más largo (179 minutos frente a 121 minutos, $p=0,004$ ) en comparación con el grupo con control hiliar renal. No hubo diferencia significativa en la creatinina sérica postoperatoria $(1,26 \mathrm{mg} / \mathrm{dl}$ frente a $1.45 \mathrm{mg} / \mathrm{dl}$, $\mathrm{p}=0,075$ ) entre los grupos. Concluyeron que el pinzamiento del hilio renal durante la resección renal y la renorrafia parecen asociarse a menor pérdida de sangre y tiempos quirúrgicos laparoscópicos más cortos.

Hemos evaluado los resultados de la heminefrectomía (escisión de $\geq 30 \%$ del parénquima renal) laparoscópica en 41 pacientes con tumor renal y hemos comparado los datos de resultados con los de una cohorte contemporánea sometida a NPL para tumores más pequeños ${ }^{19}$. El grupo de heminefrectomía laparoscópica tenía tumores más grandes $(4,0 \mathrm{~cm}$ frente a $2.4 \mathrm{~cm}, \mathrm{p}<0,001)$ con mayor extensión intraparenquimatosa $(2,3$ $\mathrm{cm}$ frente a 1,4 cm, p<0,001). Además, la heminefrectomía laparoscópica se asoció a un tiempo de isquemia caliente más largo $(38,7$ frente a 34,2 $\min , p=0,01)$. No hubo diferencias significativas entre los 2 grupos en lo que se refiere a pérdida de sangre, complicaciones intraoperatorias y complicaciones postoperatorias.

En un intento por mejorar la hemostasia, se ha estudiado el uso de agentes auxiliares. Nuestro grupo ha comparado retrospectivamente los datos de resultados en 131 pacientes sometidos a nefrectomía parcial laparoscópica con $(\mathrm{N}=63)$ o sin el uso de FloSeal $(\mathrm{N}=68)^{20}$. Ambos grupos eran comparables en lo que se refiere a características del tumor y quirúrgicas. El grupo que usó el sellante hemostásico biológico tuvo significativamente menos complicaciones y tendió hacia una menor incidencia de complicaciones hemorrágicas.

Con experiencia, pueden realizarse casos más difíciles con el acceso laparoscópico, incluidos los tumores hiliares y centrales y los tumores situa- 
dos en riñones únicos. Los autores han publicado NPL en 25 pacientes con tumores hiliares ${ }^{21}$ (definidos como tumores situados en el hilio renal, en los que se demostrara que estaban en contacto físico con la arteria y/o la vena renal en la TC preoperatoria) con un tamaño tumoral medio de $3,7 \mathrm{~cm} \mathrm{(1-10,3),} \mathrm{isquemia} \mathrm{caliente}$ media de 36,4 min (27-48), pérdida estimada de sangre de 231 cc (50-900), tiempo quirúrgico de $3,5 \mathrm{~g}(2-5)$ y estancia hospitalaria media de 3,5 días $(1,5-6,7)$. No se observaron conversiones a cirugía abierta y se produjeron complicaciones en 5 pacientes (3 sangrados, 1 fibrilación auricular y 1 neumonía). En estos casos, la escisión tumoral se inició siempre a lo largo de su borde parenquimatoso lateral y avanzó en una dirección de lateral a medial, para evitar la lesión de vasos importantes. En una comparación contemporánea de la NPL para los tumores de localización central frente a los periféricos, realizada en nuestro centro ( $\mathrm{n}=154$ y 209 , respectivamente), las tasas de complicaciones no fueron significativamente diferentes entre ambos grupos. En nuestra serie de NPL en tumores situados en riñones únicos $(n=$ 22), la media del tiempo de isquemia fue de 29 min (14-55), el porcentaje de riñón extirpado fue del 23\% (10-65), con 3 complicaciones importantes (lesión ureteral, sangrado y embolismo pulmonar). Los resultados funcionales mostraron un aumento de la creatinina sérica del 33\% (de 1,2 a $1,5 \mathrm{mg} / \mathrm{dl}$ ) y disminución de la tasa de filtración glomerular estimada del $27 \%$ (de 67,5 a 50 $\mathrm{ml} / \mathrm{min} / 1,73 \mathrm{~m}^{2}$ ), ambos directamente proporcionales a la cantidad de parénquima extirpado.

\section{Resultados oncológicos}

Presentamos nuestra serie con un seguimiento intermedio, con 100 pacientes y cada uno con un seguimiento mínimo de 3 años, con resultados comparables a las series contemporáneas de nefrectomía parcial abierta. La supervivencia global y específica de cáncer fueron del 86\% y el 100\%, respectivamente. Se encontró un margen positivo focal para $\mathrm{CCR}$, pero en ningún paciente hubo pruebas de recidiva local o en el lugar del puerto.

\section{CONCLUSIONES}

La NPL es una opción de tratamiento emergente, eficaz, para pacientes seleccionados, con resultados oncológicos a un plazo intermedio comparables a los de la nefrectomía parcial abierta. Estamos ampliando nuestras indicaciones para incluir tumores que son más grandes, muy infiltrantes, que se presentan en localizaciones menos favorables técnicamente (hiliares, centrales) y en pacientes seleccionados con riñones únicos. Sin embargo, la NPL sigue siendo una operación difícil, que deben realizar cirujanos con experiencia en procedimientos laparoscópicos urológicos avanzados.

\section{REFERENCIAS}

1. McDougall EM, Clayman RV, Chandhoke PS, Kerbl K, Stone AM, Wick MR, et al. Laparoscopic partial nephrectomy in the pig model. J Urol. 1993;149(6):1633-1636.

2. Winfield HN, Donovan JF, Godet AS, Clayman RV. Laparoscopic partial nephrectomy: initial case report for benign disease. J Endourol. 1993;7(6):521-526.

3. Janetschek G, Jeschke K, Peschel R, Strohmeyer D, Henning K, Bartsch G. Laparoscopic surgery for stage T1 renal cell carcinoma-radical nephrectomy and wedge resection. Eur Urol. 2000;38(2):131-138.

4. Gill IS, Matin SF, Desai MM, Kaouk JH, Steinberg A, Mascha E, et al. Comparative analysis of laparoscopic versus open partial nephrectomy for renal tumors in 200 patients, J Urol. 2003;Jul;170(1):6468.

5. Kim FJ, Rha KH, Hernandez F, Jarrett TW, Pinto PA, Kavoussi LR. Laparoscopic radical versus partial nephrectomy - assessment of complications. J Urol. 2003;170(2 Pt 1):408-4011.

6. Simon SD, Ferrigni RG, Novicki DE, Lamm DL, Swanson SS, Andrews PE. Mayo Clinic Scottsdale experience with laparoscopic nephron sparing surgery for renal tumors. J Urol. 2003;169(6):2059-2062.

7. Hoznek A, Salomon L, Antiphon P, Radier C, Hafiani M, Chopin DK, et al. Partial nephrectomy with retroperitoneal laparoscopy, J Urol. 1999 Dec;162(6):1922-1926.

8. McDougall EM, Elbahnasy AM, Clayman RV. Laparoscopic wedge resection and partial nephrectomy - the Washington University experience and review of the literature, JSLS. 1998;2(1):15-23.

9. Rassweiler JJ, Abbou C, Janetschek G, Jeschke K. Laparoscopic partial nephrectomy The European experience. Urol Clin North Am. 2000 Nov;27(4):721-736

10. Gill IS, Desai MM, Kaouk JH, Meraney AM, Murphy DP, Sung GT, et al. Laparoscopic partial nephrectomy for renal tumor - duplicating open surgical techniques, J Urol. 2002;167(2 Pt 1):469-467;

11. Licht MR, Novick AC. Nephron sparing surgery for renal cell carcinoma. J Urol. 1993 Jan;149(1):1-7.

12. Gill IS, Abreu SC, Desai MM, Steinberg AP, Ramani AP, Ng $\mathrm{C}$, et al. Laparoscopic ice slush renal hypothermia for partial nephrectomy: The initial experience. J Urol. 2003; 170(1):52-56.

13. Novick AC. Renal hypothermia - in vivo and ex vivo, Urol Clin North Am. 1983;10(4):637-44.

14. Janetschek G, Abdelmaksoud A, Bagheri F, Al-Zahrani H, Leeb K, Gschwendtner M. Laparoscopic partial nephrectomy in cold ischemia: renal artery perfusion. J Urol. 2004;171(1):68-71. 
15. Landman J, Venkatesh $R$, Lee $D$, Vanlangendonck $R$, Morissey K, Andriole GL, et al. Renal hypothermia achieved by retrograde endoscopic cold saline perfusion: technique and initial clinical application. Urology. 2003;61(5): 1023-11025.

16. Gill IS, Matin SF, Desai MM, Kaouk JH, Steinberg A, Mascha E, et al. Comparative analysis of laparoscopic versus open partial nephrectomy for renal tumors in 200 patients. J Urol. 2003;170(1):64-68.

17. Desai MM, Gill IS, Ramani AP, et al. Impact of warm ischemia on renal function after laparoscopic partial nephrectomy. J Urol (Submitted)

18. Guillonneau B, Bermudez H, Gholami S, El Fettouh H, Gupta R, Adorno Rosa J, et al. Laparoscopic partial nephrectomy for renal tumor: single center experience comparing clamping and no clamping techniques of the renal vasculature. J Urol. 2003;169(2):483-486.

19. Finelli A, Gill IS, Desai MM, Tam YH, Moinzadeh A, Singh D, et al. Laparoscopic heminephrectomy for tumor. Urol. (in press).

20. Gill IS, Ramani AP, Spaliviero M, Xu M, Kaouk JH, Desai MM. Improved hemostasis during laparoscopic partial nephrectomy using gelatin matrix thrombin sealant. J Urol (submitted).
21. Gill IS, Colombo JR Jr, Frank I, Moinzadeh A, Kaouk J, Desai M. Laparoscopic partial nephrectomy for hilar tumors. J Urol. 2005;174(3):850-853.

22. Frank I, Colombo JR Jr, Rubinstein M, Desai M, Kaouk J, Gill IS. Laparoscopic partial nephrectomy for centrally located tumors. J Urol. 2006;175(3 Pt 1):849-852.

23. Gill IS, Colombo JR Jr, Moinzadeh A, Finelli A, Ukimura O, Tucker K, et al. Laparoscopic partial nephrectomy in solitary kidney. J Urol. 2006;175(2):454-458.

24. Moinzadeh A, Gill IS, Finelli A, Kaouk J, Desai M. Laparoscopic partial nephrectomy: 3-year follow-up. J Urol. 2006 Feb;175(2):459-462.

Dr. Inderbir S. Gill

Sección de Cirugía Laparoscópica y Robótica

Glickman Urological Institute

The Cleveland Clinic Foundation

9500 Euclid Avenue, A100

Cleveland, $\mathrm{OH} 44195$

E-mail: gilli@ccf.org 